\title{
Melting n-eicosane in scaled-up model of capsule for use in hot climate thermal-comfort garments
}

\author{
Václav Tesař ${ }^{1, *}$, Jozef Kordík ${ }^{1}$
}

${ }^{1}$ Institute of Thermomechanics v.v.i., Czech Academy of Science, Dolejškova 5, 18200 Praha-Kobylisy, Czech Republic

\begin{abstract}
Recent ideas in development of garments for thermal comfort in desert climates concentrate on textile containing small capsules filled with material melting to store latent heat at roughly human body temperature. This cools the garment wearer during the hot day - and later keeps him warm in the cold desert night. This article investigates the melting process in a single scaled up model of spherical capsule filled with n-eicosane - a material melting at $\mathrm{T}_{\mathrm{m}}=35.7^{\circ} \mathrm{C}$. In the experiment, the capsule model was heated starting from the initial temperature $30^{\circ} \mathrm{C}$ by warm water at $50.4^{\circ} \mathrm{C}$. The large scale of the model made possible study of details of the melting process.
\end{abstract}

\section{Introduction}

The main problem with energy in general is the lack of balance between supply and demand. Solution is sought in energy storage. The simplest among the storage principles is heating a mass of material when the energy is abundant and later, in the time of demand, letting it cool down to recover the energy. This heating method is not particularly attractive at industrial large scales because in most applications the energy is required in electric and not thermal form and the conversion is not easy. There are, however, quite promising small-scale heat storage applications with the thermal form in both input and output and thus no need of conversions. Particularly attractive is the idea of garments made of "smart" textiles. They cool the wearer during day and then keep him warm in cold nights [1] with melting and solidification using the latent heat, without substantial changes of temperature. The phase change material is in the textile kept in small capsules. In this article, the melting process inside capsules is studied using a scaledup model.

\section{1 Earlier garment ideas}

Cooling and heating garments are not uncommon in the aeronautical and space engineering, with their wearers inside or near to a vehicle containing the temperature keeping system. The present goal is different: an autonomous garment worn by persons walking in hot environments on foot and physically active. The key acceptance factor are thus small size and weight. If the system involves a fluid flow, then for its generation has to be carried a driving power source (e.g., a motor and a battery) sufficient for a mission lasting several hours. The standard approach to the developments so far has been the refrigerator cycle principle - with miniaturisation based on ideas from microfluidics [1]. A simple calculation, however, indicates that the resultant size and weight of the garment based on these ideas would make the wearing very inconvenient. The fundamental problem faced by the garment designer is the basic law of heat flow, always directed from the body at higher temperature to a cooler one. This means the classical cooling necessitates some form of heat-pump machinery. for compression and expansion of the working fluid to generate the needed temperature difference. Weight, the critical acceptance factor, favours air as the working fluid. Liquids, despite their better heat transfer properties, are too heavy.

\section{2 Earlier idea of reversed Brayton cycle}

In the systems proposed so far, heat was removed from the human body by the air flow in the tubes inside the textile material, Fig. 1. Upon leaving the tubes a, Fig. 2., the air is compressed, from (1) to (2), This increases its temperature above that of the ambient outer atmosphere

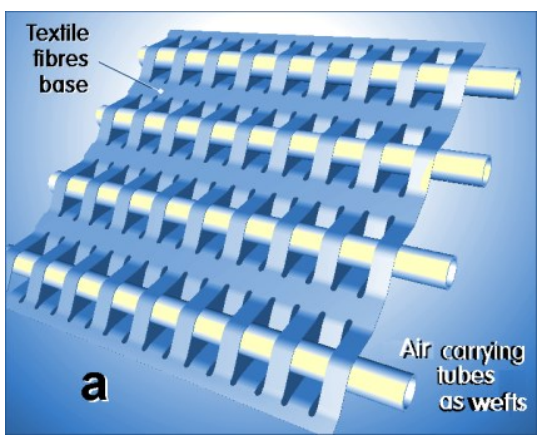

Fig. 1. Earlier idea of cooling textile. Interwoven small tubes for flow of air cooled by expansion.

*Corresponidng author: tesar@it.cas.cz 


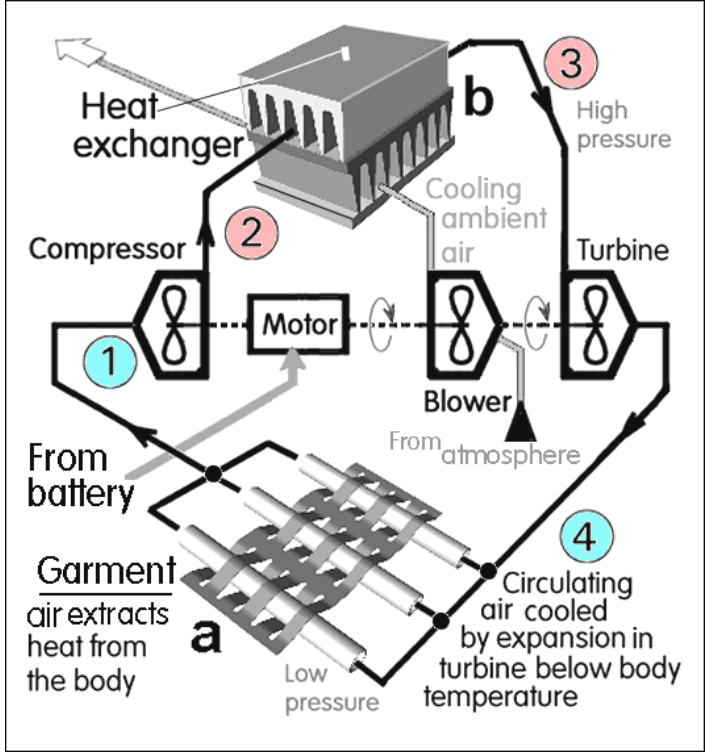

Fig. 2. Schematic presentation of the cooling garment idea as under development so far [1]. The air flow after expansion from (3) to (4) is fed into the tubes in the textile a where it removes heat from the garment wearer's body. The air temperature is then increased by compression (1) to (2), making possible removal of heat from the air in the exchanger $\mathbf{b}$.

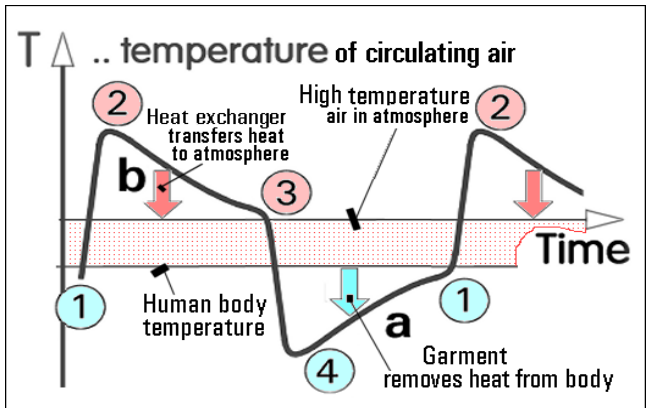

Fig. 3. The time dependence of air temperature according to Fig. 2. After being cooled by expansion in (3)-(4), the air in $\mathbf{a}$. between (4) and (1) absorbs heat from wearer's body. It is then heated by compression to discard the heat between (2) and (3).

- so that the heat from the circulating air may be removed from the system in the exchanger $\mathbf{b}$. The air flow undergoes repetitive cycles as shown in Fig. 3. The expansion turbine shown there cannot deliver sufficient power to drive the compressor and blower in this reversed Brayton cycle [2]. Thus it needs driving of the system by an external power source. Design of rotating machines of acceptable size and weight is itself one of the main difficulties. The motor shown in Fig. 2 assumes powering by a carried battery, another significant addition to the system weight.

\section{New idea: cooling (as well as heating) by phase changing material}

A much more promising solution of the cooling garment problem is offered by the latent-heat storage principle [3] - with the advantage of no rotating machinery and operation without the high temperature differences. The used processes are passive, i.e. there is no need a power supply from an external source (like the batteries in Fig. 2). What is necessary, however, is a periodically varying ambient temperature - which is in deserts available as the alternating periods of hot day and cool night. The "smart" textile from which the garment is made contain small capsules, typically of sub-millimetre size (diameter less than $1 \mathrm{~mm}$ ). They may be held captive in chambers as shown in the example presented in Fig. 4 - or simply distributed between the textile fibres and fixed to them. The critical decision factor is the choice of the heat absorbing material inside the capsules. It must melt at or near human body temperature $\mathrm{T}_{\mathrm{m}}$. Later, in cold desert night at the atmosphere temperature below $T_{m}$, this material solidifies and its latent heat is released to keep the wearer conveniently warm.

The amount of the phase-changing material for a given wearer's mission duration depends on its specific latent heat $e_{L}[\mathrm{~J} / \mathrm{kg}]$. This, as discussed in [3] is the second material property that has to be considered in the choice of the material. As discussed in [3]. there are three groups of the materials that offer acceptable properties. In the experiment discussed below in this article, yet another factor considered. It was the variation between opaque solid and transparent liquid. Of course, this is of no importance for the operational use and was convenient for photographic recording of the melting process and evaluation of the solid fraction inside the capsule.

In spite of the differences between the two approaches - the air flow Brayton cycle Fig. 2 and the phase-change Fig. 4 - it should be noted that there is a certain topological equivalence in the gross aspects of both operation modes, apparent from the comparison of Figs. 3 and 5. The durations of the two heating/cooling cycles is very much different. It is merely minutes or less in the former case and diurnal atmospheric temperature variations between the DAY and NIGHT regimes. Also the temperature levels are different. In the former case the state (2) the temperature at the exchanger inlet must be made quite high to accommodate the gradual decrease towards the following state (3) still above the atmosphere - while the latent heat melting process is isothermal so that the daytime temperature just above $\mathrm{T}_{\mathrm{m}}$.

Of course, in order to avoid the obvious objections by the potential wearers against any weight to be carried in addition to the normal load (itself unpleasant in a hot day) - it is necessary to minimise the size and weight. A rational minimisation depends critically on available

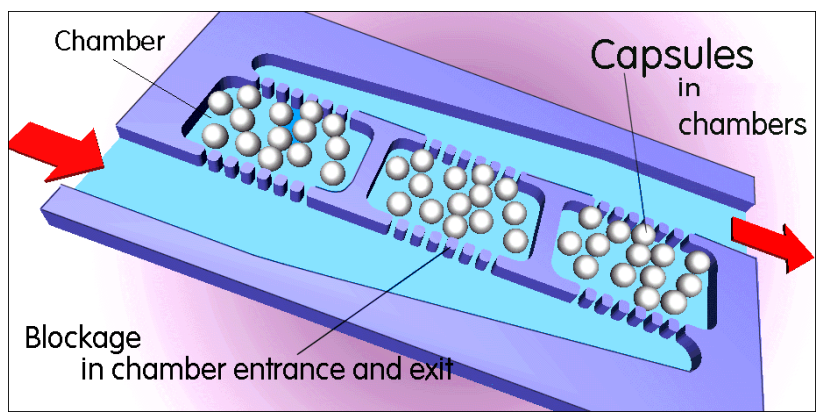

Fig. 4 Detail of one of the channels in the textile material with sub-millimetre size capsules for heating and cooling by latent heat input and removal into and from air flow through the textile pores (in principle not strictly necessary, but useful for removal of the wearer's perspiration humidity and increase the heat transport intensity). 


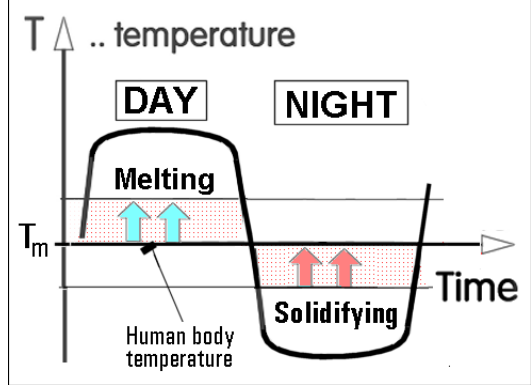

Fig. 5. Although the mutual connection between the system shown in Fig. 2 and the "smart textile" idea in Fig. 4 may be not apparent, the comparison of the time-dependence diagrams Fig. 3 and. 5 shows a certain underlying commonality of the processes.

sound knowledge of the phase-change material properties and details of its behaviour during the melting/ solidifying processes inside the capsule. Since the idea of the thermally comfortable textile is relatively new, the extent of knowledge accumulated so far by involved researchers is not sufficient. Information in available literature, such as, e.g., the data listed in [3], are far from complete. Typical case is the absence of knowledge about the character of the convective motions inside the melt. Of course, some data are sensitive due to the potential military uses, nevertheless at least the basic physics deserves a useful discussion.

\section{Melting process}

\subsection{Motion of the remaining solid}

At the very beginning of the melting process, it is assumed that the whole internal spherical space of the capsule is filled by the heat storage material in its solid state. The latent heat of melting is transported from outside across the capsule shell walls. Thus the heat transfer is obviously very much dependent also on the external conditions outside the shell. For investigations of these outer shell phenomena is currently built a dedicated wind tunnel for variable outer air flow. Initial information about it is described in ref. [3].

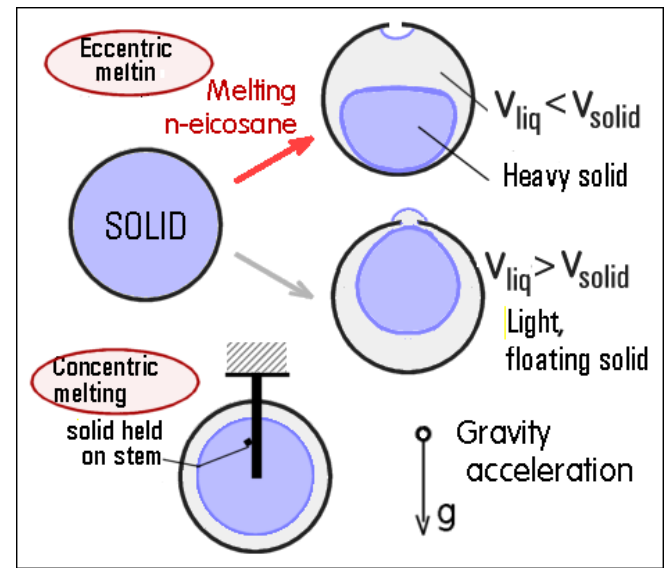

Fig. 6. Vertical positions the solid-state phase not yet molten material inside the spherical shell depends on the change of the specific volume during the melting. In the experiments, the $\mathrm{n}$ eicosane in its liquid phase is lighter than the solid which thus falls to the shell bottom - unless it is fixed to a stem.
Attention in the present article is focused on the internal processes within the capsule. They are dominated by the difference between the specific volumes $v\left[\mathrm{~m}^{3} / \mathrm{kg}\right]$ : - that of the solid phase, $\mathrm{V}_{\text {solid, }}$ and the liquid phase $\mathrm{V}_{\text {liq. }}$. As represented schematically in Fig.6, in the case of the experiment with the $n$-eicosane, the liquid phase is lighter so that the not yet molten solid fraction falls to the shell bottom. Obviously, theoretical analysis of this process, three-dimensional with the eccentric position of the solid body, is quite difficult. It may be therefore useful to simplify the study at the beginning by fixing the solid phase body to a stem securing the concentric position leasing to two-dimensional planar geometry problem. If the stem is small, its presence is unlikely to influence the melting process to any significant degree.

Another phenomenon also presented in the schematic pictures shown in Fig. 6, associated with the specific volume change $\mathrm{V}_{\text {liq }}>\mathrm{V}_{\text {solid, }}$, is the expansion of the liquid. In the illustration it is assumed that the phase changing material was filled - in the initial liquid state - into the capsule from above through a top inlet left open. In this case the thermal expansion $V_{\text {liq }}>V_{\text {solid }}$ leads to some small amount of the liquid escaping from the shell (as indicated by the small drop on the shell top in Fig. 6). In the other $\mathrm{V}_{\text {liq }}<\mathrm{V}_{\text {solid }}$ case, of main interest in this article, the volume contraction results in the surface of the liquid gradually moving downwards, away from the shell top, thus leaving a (very small) part of the capsule filled with air.

\subsection{Convective streaming}

Apart from the slow motion of the solid/liquid boundary during the melting process presented in Fig. 6, the internal processes inside the spherical shell may be much more complicated. The reason are the convection streaming flows in the molten liquid caused by the specific volume changes and the resultant lift forces.

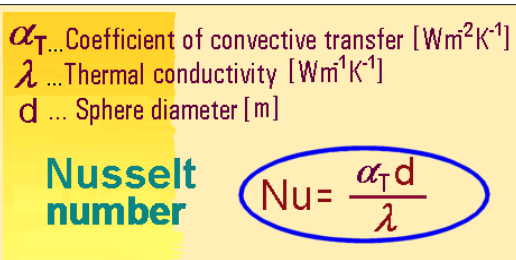

Fig. 7. Intensity of heat transfer by convection is nondimensionalised by comparing it - in the magnitude of Nusselt number - with the conduction heat transfer

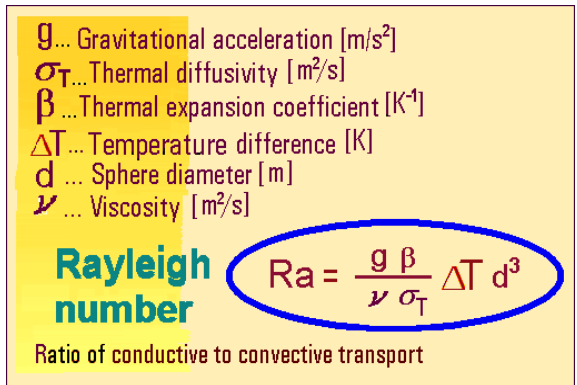

Fig. 8. The non-dimensionalised intensity of the vertical force acting on the liquid which generates the convective streaming is represented by the magnitude of Rayleigh number Ra. 
As in other cases of convection heat transfer, the streaming increases the transfer intensity from the solid walls. This thermal transport is much efficient than conduction through stationary, non-moving material. In the present case of latent heat, the thermal difference effects between internal locations are, of course, smaller than in many other natural convections, but they are present and must not be neglected.

The results of the study presented in this article assume that the convective flows cause the same or analogous effect in the microcapsule of sub-millimetre size and in the very much scaled up model used in the laboratory tests. This means the thermomechanical conditions in both must be identical. It is ensured when there is the same value of the Nusselt number, the nondimensional factor defined in Fig. 7. The effect driving the streaming, depending on the expansion in the gravitational field, and therefore depends on the quantities listed in Fig. 8, the definition of the Rayleigh number Ra. .

A typical mutual dependence between the two nondimensional factors $\mathrm{Nu}$ and $\mathrm{Re}$, evaluated from existing literature for the non-melting case $[4,5], \mathrm{s}$ here shown in Fig. 9. This particular case was evaluated from experiments performed in air with invariant geometry of the two spherical boundaries (no melting but just heat transfer). The geometry shown in this diagram is characterised by the ratio 2.0 of the outer shell surface diameter $d_{0}$ to the inner solid sphere of diameter $d_{i}$. It was the inner spherical surface that was heated in this experiment. It is not necessary to emphasise that for a different geometry ratio do / di the Nusselt number value at the same Rayleigh number is different. Thus in the present case with melting, even if it could be accepted that the solid not yet melted body inside the capsule is spherical (which, of course, it is not because the intensity of melting varies across the body surface) it should be necessary for each time instant of the experiment to evaluate a diagram similar to Fig. 9. Of course, if this were an experimental investigation, the total amount of the extracted and evaluated information would be extremely large. Also, in the case of the melting the thermal transport is accompanied - and may be substantially influenced - by a diffusion transport. These facts thus provide one of the explanations why the investigated problem of melting is still in need of the studies such as those discussed in the present article. The difficulties associated with the measurements of the dependence between $\mathrm{Nu}$ and $\mathrm{Ra}$ at each time are simply too large. Nevertheless, it may be expected that at any instant of time in the laboratory test the dependence $\mathrm{Nu}=$ $f(\mathrm{Ra})$ would be topologically similar (though, of course, not quantitatively) to the diagram shown here in Fig. 9. What is immediately apparent in this diagram are two different behaviour regimes - above and below the critical $\mathrm{Ra}$ value. In both regimes the dependence when plotted in the logarithmic co-ordinates follows the fitted straight line.

The extrapolated two straight lines intersect in the point of nominal critical state Crit. At the left-hand side, i.e. at Reyleigh number values below $\mathrm{Ra} \sim 30 \quad 000$, the heat transfer is characterised by Nusselt number

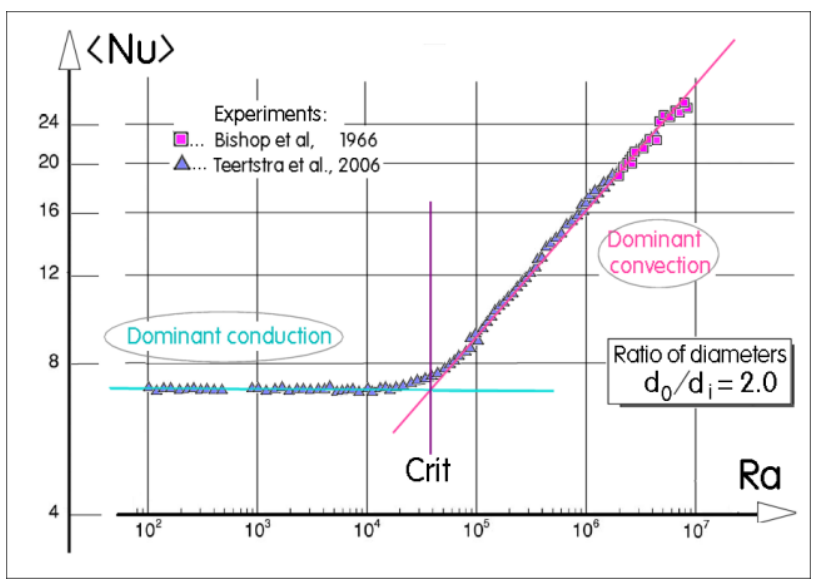

Fig. 9 Typical dependence of heat transfer intensity on Rayleigh number $\mathrm{Ra}$ in conditions existing in the space between concentric spheres - adapted from [4, 5]. The convective streaming driven by buoyancy is present at $\mathrm{Ra}$ above the nominal critical state Crit.

constancy. This is a consequence of conductive character of the heat transport. Obviously, in these regime the viscous friction inside the liquid is so strong that the liquid is practically immovable. On the other hand, at $\mathrm{Ra}>30000$, the larger and increasing Nusselt number values indicate the dominance of the convection. The larger is $\mathrm{Ra}$ there, the stronger is the convective flow and the more efficient is the heat transfer indicated by the higher $\mathrm{Nu}$.

When the information obtained in the experiment discussed by the present authors is evaluated, the key factor in the processing will be the identification of the nominal critical state value Crit - and thus finding at what side (convective at right or conductive at left) are the Ra values. They should be, of course, the same both in the actual capsules and in the scaled-up model.

It should be also mentioned that the parameters $\mathrm{Nu}$ and $\mathrm{Ra}$ are defined in Figs. 7 and 8 locally, i.e. at a particular point on the surface of the shell. These values vary from point to port on the surface of the solid fraction body.. The values actually plotted in the evaluation of the experimental data, such as in Fig. 9, will be the ensemble averages $<\mathrm{Nu}>$ (and also $<\mathrm{Ra}>$ ) over the whole set of the individual surface data points.

\subsection{Character of streaming}

There is already an existing literature, e.g. [6], presenting computational results about the character of the convective streaming flowfield generated by fluid thermal expansion and consequent appearance of lift forces. It is expected that in

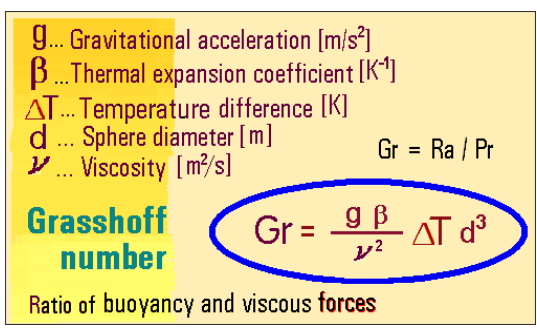

Fig. 10. Character of the convection streaming driven by the buoyancy is determined by the magnitude of Grasshoff number $\mathrm{Gr}$ (closely related to Ra as defined in Fig 8). 
further development of the discussed investigations the pathlines in the flowfield will find this information useful again, not forgetting the fact that the existing literature data are based on the invariance of the geometry (which, of course, varies in the case of melting), and ate less efficient in the constant-temperature latent heat case. The typical example of the natural convection flowfield, adapted from. [6], is the diagram in Fig. 11. The flow there took place was in air inside the annular space between two concentric spherical walls with electric heating of the inner sphere. It should be noted that this is yet another difference from the case discussed by the present authors where it is the other, outer spherical wall that is heated. The geometry of the flow configuration is in Fig. 11 characterised by the (constant) ratio $d_{o} / d_{i}=2.17$ of the outer surface diameter $d_{0}$ to the inner heated solid sphere of diameter $d_{i}$. This value of the ratio is very near to the that in the concentric case experiment Fig. 9. The planned experiments, of course, have to acquire information for the gradual change of size and shape of the solid body (i.e. the case of the gradual increase of the ratio $\left.d_{0} / d_{i}\right)$ - and again with the geometry of the melting interface much more complex than the spherical surface fully described by a single numerical value).

Of significant importance in the present discussion is the suitable dimensionless parameter that provides (together with the geometry) the instantaneous overall characterisation of the convective streaming flows. This parameter is not, as might be perhaps expected, the Rayleigh number of Fig. 8, but, instead, the very closely related Grasshoff number Gr, defined in Fig. 10. The pathlines presented in the example shown in Fig. 11 were computed in [6] for the conditions characterised by the large Grasshoff number value (near $\mathrm{Gr} \sim 10^{6}$ ) at which the generated flows are unsteady, varying in time.

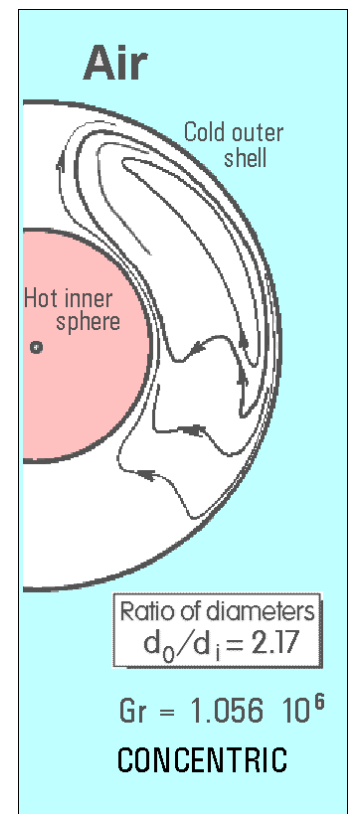

Fig. 11. An example of convection streaming trajectories between concentric spheres, of which the inner one is heated. The pathlines (streamlines do not exist in this case) shown here were obtained by adapting the photographs presented in [6]. Note that shown here is the case of Grasshoff number Gr of the order. This is a value at which the streaming is unsteady and its trajectories vary in time.

\subsection{Effect of eccentricity}

The cases discussed in the previous Sects.3.1 to 3.3 are simplified by assuming concentric position of the internal spherical surfaces. This makes easier numerical solution as well as potential analytic computations. It does not, however, correspond to reality. In the model experiment, the concentricity is obtained by attaching the solid molten body at a stem (as mentioned in association with the illustration in the bottom part of Fig. 6). No such stem, of course, is inside the actual microcapsules of the smart textile. There the solid-fraction body during the melting process always falls down in the direction of the external acceleration (mostly the gravity acceleration as indicated in the top part of Fig. 6).

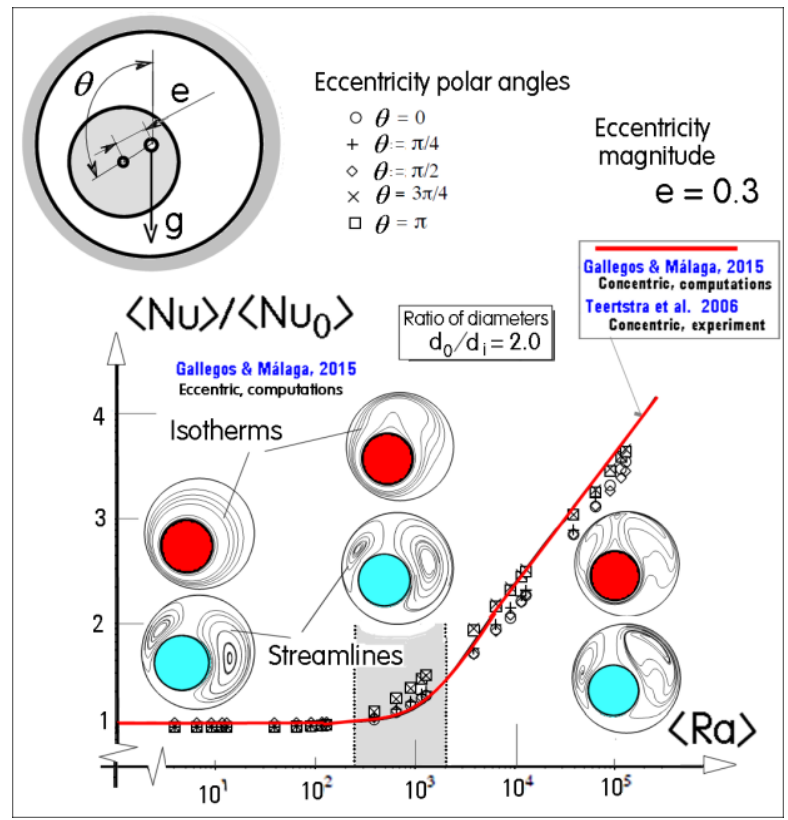

Fig. 12. Computations adapted from ref. [ ] of velocity and temperature fields between two spheres in the eccentric configurations. In the cases of interest for the present article the polar angle between eccentricity $\mathrm{e}$ and the graviational acceleration (Fig. 6) is solely $\theta=\pi$. At any rate, these results demonstrate that the deviations from the concentric case Fig. 9 are rather small.

In literature, e.g. [6], are available results of numerical flowfield and temperature solutions. For the excentric configurations. Example computation results data from ref. [6] are here presented in Fig. 12. analogous to Fig. 9. While in Fig. 9 the Nusselt numbers are the absolute values obtained in the experiment, in Fig. 12 the values on the vertical axis are relative - related to the value $\left\langle\mathrm{Nu}_{0}\right\rangle$ approached asymptotically at very small $\mathrm{Ra}$ in the left-hand side of the diagram. Fortunately, despite the complexity, the deviations from the concentric cases are in Fig. 12 rather small.

\subsection{Elasticity of the shell}

The experimental configuration with the opening for air on the top of the capsule, making possible free expansion or volumetric contractions of the phase change material as shown in Fig. 6 is, of course, not acceptable for the smart textile as it would mean leak of the material in its molten phase. While it was used in the initial tests, 


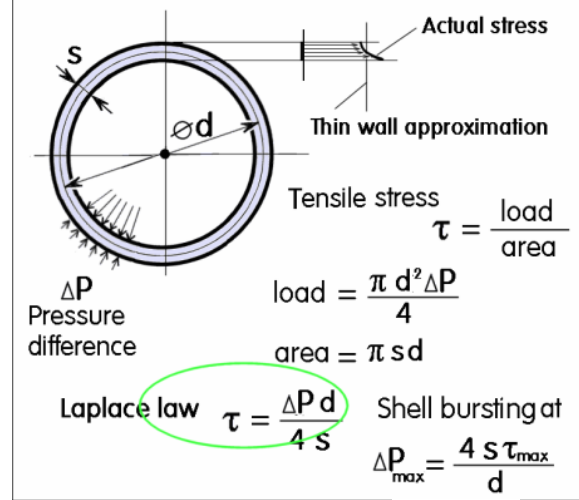

Fig. 13. Thermal expansion of the $V_{\text {liq }}>V_{\text {solid }}$ phase-change materials causes in the walls of closed-volume shell a tensile stress which may reach the strength limit of the shell material, which then bursts open.

at a later stage of the program it is necessary for the capsule model to be fully closed. Modelling of the he encapsulated material behaviour will involve shell deformations. There is an extremely wide range of the useful shell materials for the microcapsules corresponding to the glass in the scaled up model. The choice will be mainly dictated by the chosen method of the microcapsule shell manufacturing. Some of the potential materials might be of organic character. Typical for them are rather complex mechanical deformation properties. To begin with a less difficult problem, in introductory discussion in Section 3.5 will be assumed Hookean material, i.e. with linear deformation properties. When deformed by the change $\Delta \mathrm{V}$ of the internal volume

$$
\mathrm{V}=\pi \mathrm{d}^{3} / 6
$$

of the fully closed capsule, the phase change material in it will respond by the pressure difference $\Delta \mathrm{P}$ acting between the outer and inner surface of the shell. The assumption of the Hookean linearity means

$$
\Delta \mathrm{P}=\Delta \mathrm{V} / \mathrm{C}
$$

- where $C\left[\mathrm{~m}^{4} / \mathrm{N}\right]$ is the compliance of the capsule. The larger is its numerical value, the smaller is the pressure increase. The stress and strains are governed by the law for the tensile stress $\tau$ as a function of the relative change of the vessel diameter $d$

$$
\tau=\mathrm{E} \Delta \mathrm{d} / \mathrm{d}
$$

... where $E\left[\mathrm{~N} / \mathrm{m}^{2}\right]$, the Young's modulus, is the property of the particular shell material.

Approximate values of $E$ - the slopes of the linear relations between stress and strain - may be estimated by comparison with the values of the several well known materials in Tab. I.

Tab I

E

$\left[\mathrm{N} / \mathrm{m}^{2}\right]$

$\begin{array}{lc}\text { Rubber } & 7 \\ \text { Cartilage } & 24 \\ \text { Tendon } & 600\end{array}$

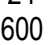

1

$\begin{array}{lc}\text { Bone } & 21000 \\ \text { Glass } & 70000 \\ \text { Steel } & 210000 \\ \text { Diamond } & 1200000\end{array}$

It is plausible in the cases of interest here to use the thin wall approximation and then to derive in Fig. 13 by simple evaluations of the force load and the area on which this load acts. The result is the Laplace law for tensile stress $\tau$.

The expression for the volumetric deformation $\Delta \mathrm{V}$ is obtained by simple differentiation of eq. (1)

$$
\Delta \mathrm{V} / \Delta \mathrm{d}=\pi \mathrm{d}^{2} / 2
$$

The expression for $\tau$ in eq. (3) is equal to $\tau$ in the Laplace law. Comparing them,

$$
\tau=\mathrm{E} \Delta \mathrm{d} / \mathrm{d}=\Delta \mathrm{P} \mathrm{d} / 4 \mathrm{Es}
$$

the change of shell diameter $\mathrm{d}$ is

$$
\Delta \mathrm{d} / \Delta \mathrm{P}=\mathrm{d}^{2} / 4 \mathrm{Es}
$$

Multiplying eq.(6) by eq. (4) leads to the expression for the compliance

$$
\mathrm{C}=\Delta \mathrm{V} / \Delta \mathrm{P}=\pi \mathrm{d}^{4} / 8 \mathrm{Es}
$$

It is evident that compliance $C$ depends dramatically upon the shell diameter $\mathrm{d}$.

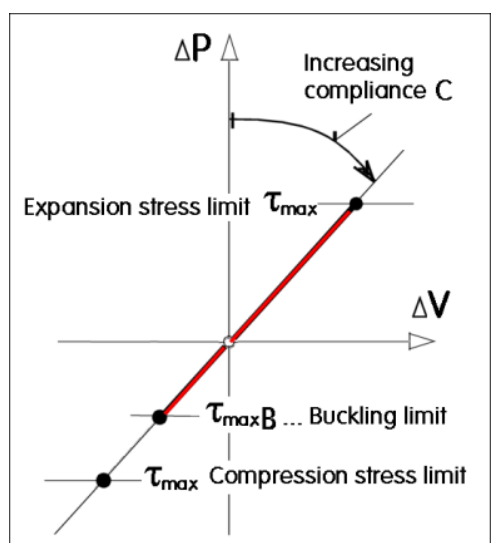

Fig. 14. Pressure difference acting on the capsule shell walls due to thermal volume change may assume a position everywhere on the red colour compliance line. This is limited by the expansion or compression maxima of the maximum tensile stress of the material.

The dependence eq. (7) is valid both for positive (expansion) and negative $\Delta \mathrm{V}$ (compression) as shown in Fig. 14. The pressure growth, however, cannot continue infinitely. Materials posses their limiting stress value $\tau_{\max }$ on both positive and negative side, Fig. 14. Approximate values of $\tau_{\max }$ at which the shell is torn into parts may by estimated by interpolation among known materials in Tab. II.

Tab II

\begin{tabular}{lc}
\multicolumn{1}{c}{$\tau_{\max }$} & {$\left[\mathrm{N} / \mathrm{m}^{2}\right]$} \\
\hline Cartilage & 3.0 \\
Human skin &
\end{tabular}

Tendon

Glass

Human hair

Nylon 
There is yet another limit apart from that in Fig. 13. When the increasing the negative tensile stress $\tau$ in Fig. 14 (decreasing the volume by the melting) upon reaching the buckling limit value $\tau_{\max }$ the shell would buckle (Figs. 14, 15) and collapse. The shell wall would cease to be perfectly spherical and exhibits a periodic series of deflections $[7,8,9]$. This also means loss of its ability to withstand the acing pressure difference. If the shell material is brittle, like e.g. glass, the buckling shell would burst. Due to the character of stability loss, the expressions presented in Fig. 15 may be strongly influenced by even small geometric imperfections of its shape [10].

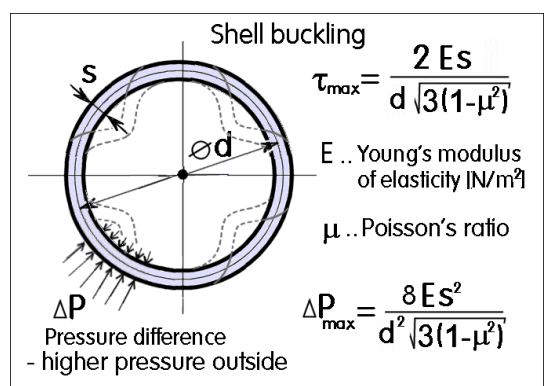

Fig. 15. When the melted material decreases its volume due to $\mathrm{V}_{\text {liq }}<\mathrm{V}_{\text {solid }}$ (as is the case shown in Fig. 6 for the n-eicosane), the shell may reach at $\tau_{\max }$ another limit, the buckling instability.

\section{Experiment}

\subsection{Materials and conditions}

Authors were able to performed a proof-of-concept experiment with the scaled-up capsule model. The experiment was laborious and time consuming (the run lasted $\sim 70$ minutes). As a result it was not possible to perform more measurement runs than the single initial one.

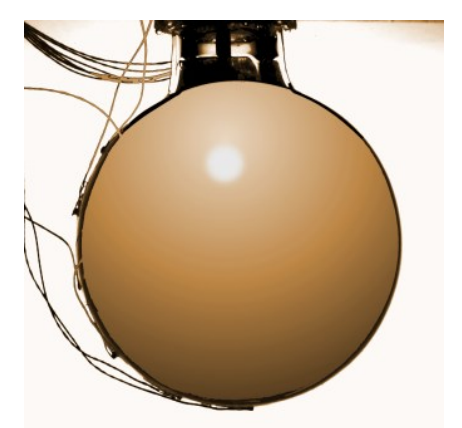

Fig. 16. Photograph of the initial stage of the experiment. The scaled-up model of the single spherical capsule, filled to the top with n-eicosane, was opaque in the solid state (while transparent when molten). Thus the solid phase fraction could be evaluated from the photographs.

As the shell was used a glass vessel obtained from manufacturer of laboratory glass components. The thin wires visible at left are leads to eight resistor-type thermometer sensors.

This was particularly regretted because the performed experimental run was originally considered unsuccessful as a consequence of the body of the solid fraction, got roughly in the middle of the test loose from the stem (sting) on which it was initially held in position. and fell to the bottom of the model capsule. However, later on when evaluating the accumulated data, it became apparent that this change in the regime provided an opportunity for learning something from both mutually different regime, as if they were launched one after another.

The phase-change material selected for this laboratory investigation - on the basis of analysis of the properties listed in [3] — was n-eicosane. The reasons behind this choice were two:

(a) The value of the melting point temperature, $\mathrm{T}_{\mathrm{m}}=$ $308.8 \mathrm{~K}=35.7^{\circ} \mathrm{C}$. It is slightly below the normal temperature of healthy human body surface, and

(b) Full transparency of the material in the molten phase. It made possible computing from photographs the fraction of the solid material within the shell. Details of mechanical and thermal properties of neicosane are presented in the three tables below. In Tab. III are the properties of the solid state, in the next Tab. IV are the properties characterising the solid-toliquid transition (and its reversal), and finally Tab. V lists the properties of n-eicosane in the liquid regime, at the temperature just above the melting $\mathrm{Tm}$. The properties were extracted from the data presented in references $[4,5,6]$.

Prior to the actual investigations, 100 millilitres of the n-eicosane phase-changing material in the molten state was filled into the model shell of outer diameter $d=$ $64.5 \mathrm{~mm}$. It was then left overnight in a refrigerator box to solidify. As the shell was used the largest sphericalglass flask from the set of four sizes available commercially from the supplier KAVALIERGLAS a.s., 28506 Sázava, Czech Republic The wall thickness of the glass sphere was $\mathrm{s}=1.5 \mathrm{~mm}$.

Photograph of the flask full of the solid solid-state neicosane material is presented in Fig. 16. In a fixed central position in the solidified solid sphere the flask was held by its filling inlet that here served in the role of the positioning stem from the top, as shown in Figs. 16, 17. The fixation, however, based on mechanical strength of n-eicosane after a certain elapsed time became loose.

By comparing the specific volumes $v\left[\mathrm{~m}^{3} / \mathrm{kg}\right]$, for the solid phase $V_{\text {solid }}=1.22710^{-3} \mathrm{~m}^{3} / \mathrm{kg}$ in Tab. I and for the liquid phase $V_{\text {lig }}=1.28210^{-3} \mathrm{~m}^{3} / \mathrm{kg}$ in Tab. III, it appears from Fig. 6 that the gradually melted body of still solid n-eicosane became loose and thereafter remained stationary, sitting on the bottom of the shell for the rest of the melting process.

Tab. III n-eicosane SOLID state properties [15]

Specific heat capacity

$1.92 \mathrm{~kJ} . \mathrm{Kg}^{-1} . \mathrm{K}$

Thermal conductivity

Specific volume

Young's modulus

Poisson's ratio

Tensile strength

$0.341 \mathrm{~W} \cdot \mathrm{m}^{-1} \cdot \mathrm{K}^{-1}$
$1.22710^{-3} \mathrm{~m}^{3} / \mathrm{kg}$
$63.210^{3} \mathrm{MPa}$
0.19
$35 \mathrm{MPa}$

$0.341 \mathrm{~W} \cdot \mathrm{m}^{-1} \cdot \mathrm{K}^{-1}$

$63.210^{3} \mathrm{MPa}$

$35 \mathrm{MPa}$ 


\section{Tab. IV n-eicosane TRANSITION properties}

$\begin{array}{ll}\text { Melting point } & 308.84 \mathrm{~K}^{1} \\ \text { Heat of melting } & 247.05 \mathrm{~kJ} / \mathrm{kg} \\ \text { Temperature of softening } & 820^{\circ} \mathrm{C}\end{array}$

Tab. V n-eicosane $\mathrm{n}$-Eicosane LIQUID properties

$\begin{array}{lc}\text { Specific heat capacity } & 0.150 \mathrm{~W}^{\mathrm{m}-1} . \mathrm{K}^{-1} \\ \text { Specific volume } & 1.28210^{-3} \mathrm{~m}^{3} / \mathrm{kg}^{-} \\ \text {Dynamic viscosity (at T = } 325 \mathrm{~K} \text { ) } & 3.1810^{-3} \mathrm{~kg} \mathrm{~m}^{-1} \mathrm{~s}^{-1} \\ \text { Coefficient of thermal expansion } & 1.0210^{-3} \mathrm{deg}^{-1} \\ \text { Kinematic viscosity (at T = } 325 \mathrm{~K} \text { ) } & 4.0810^{-6} \mathrm{~m}^{2} \mathrm{~s}^{-1}\end{array}$

\subsection{Setup}

The model capsule was in this experiment submerged in the rectangular vessel filled to the top with flowing hot distilled water, shown in Fig. 18. The vessel walls were transparent, making possible photographs of its contents.

The n-eicosane melted material was inside the largest, 100 millilitres spherical shape glass flask. It was held from the top, in the manner shown in Fig. 17, by the holder for the role of which was used the flask filling inlet. The flask was immersed into the warm water. Its constant temperature $50.44^{\circ} \mathrm{C}$ was maintained by its circulating permanently through the vessel and, outside of the vessel connected to it by hoses, through a thermostat. The flow was driven by the pump that is the inherent thermostat component. Water was flowing slowly in the test vessel in horizontal direction, from the left-hand side past the model capsule to the right-hand side. Regularity of the flowfield was achieved by the flow

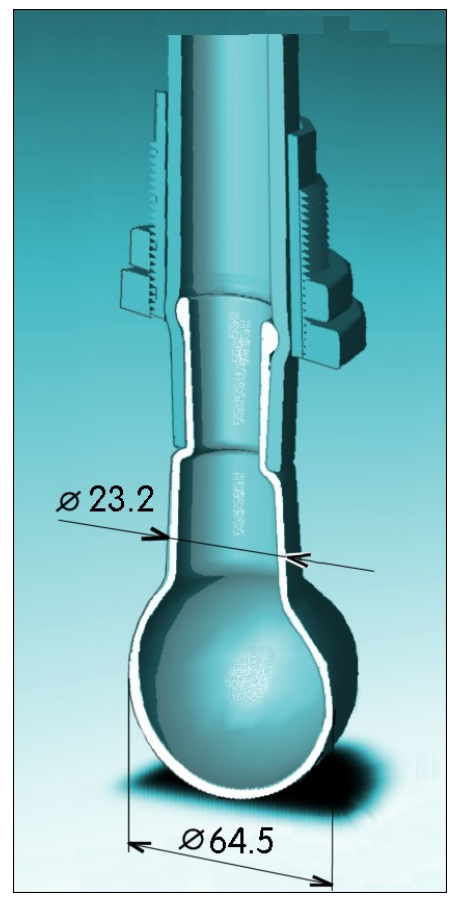

Fig. 17. The glass flask that served as the spherical capsule shell, shown here in section by meridian plane. It was held on top by the flask inlet, making possible volume changes without stressing the shell material (glass). Trade name of the glass material is SIMAX. Its mechanical and thermal properties are listed in Tab. IV. at both inlet and outlet ends through a fine sieve. Physical properties of the glass capsule model are presented in Tab. VI. Both walls of the rectangular vessel and the walls of the glass flask are in the photographs practically perfectly transparent. With the suitable choice of the background and lights thus the photographs allow precise identification of the space occupied by the not yet molten solid, as may be seen in Fig. 19 ..

Shown also in the drawing Fig. 18 are eight locations from \# 101 to \# 108 in which were positioned resistortype thermometer probe tips. As seen in the picture, probes were distributed in the surface of the spherical shell, one additional probe \# 101 was located in the centre of the sphere. The last thermometer probe \# 108 was position in the heat carrying water upstream from the capsule model.

Tab VI SIMAX glass physical material properties

\begin{tabular}{|c|c|c|}
\hline \multirow{8}{*}{\multicolumn{2}{|c|}{$\begin{array}{l}\text { Coefficient of linear thermal expansion } \\
\text { Density } \\
\text { Heat conductivity } \\
\text { Specific heat capacity }\end{array}$}} & $3.35 \times 10^{-6} \mathrm{~K}^{-1}$ \\
\hline & & $2.23 \mathrm{g.cm}^{-3}$ \\
\hline & & $1.16 \mathrm{~W} \cdot \mathrm{m}^{-1} \cdot \mathrm{K}^{-1}$ \\
\hline & & $8 \times 10^{3} \mathrm{~J} \cdot \mathrm{Kg}^{-1} \cdot \mathrm{K}$ \\
\hline & & $820^{\circ} \mathrm{C}$ \\
\hline & & $63.2 \times 10^{3} \mathrm{MPa}$ \\
\hline & & 0.19 \\
\hline & & $35 \mathrm{MPa}$ \\
\hline
\end{tabular}

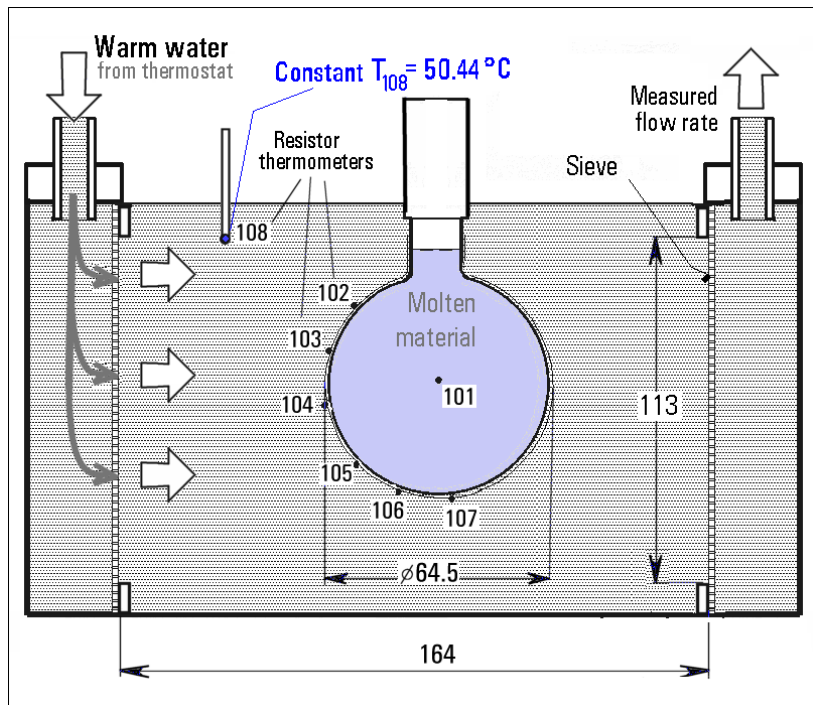

Fig. 18. Experimental set-up and its geometry. Constanttemperature water carrying the melting heat is delivered at the left-hand side through the fine vertical sieve. It flows past the tested capsule model and leaves (again through a sieve) at right, returning to the thermostat which keeps its constant temperature. The spherical model is scaled up substantially from the actual capsules to make possible detailed studies of the internal phase changes.

The distilled water entered the test setup from the thermostat at the constant temperature $\mathrm{T}_{108}=50.44^{\circ} \mathrm{C}$, i.e. significantly above the melting point temperature $T_{m}$ $=35.7^{\circ} \mathrm{C}$. Prior to the submerging into the warm water, the flask filled with the phase change material was kept 


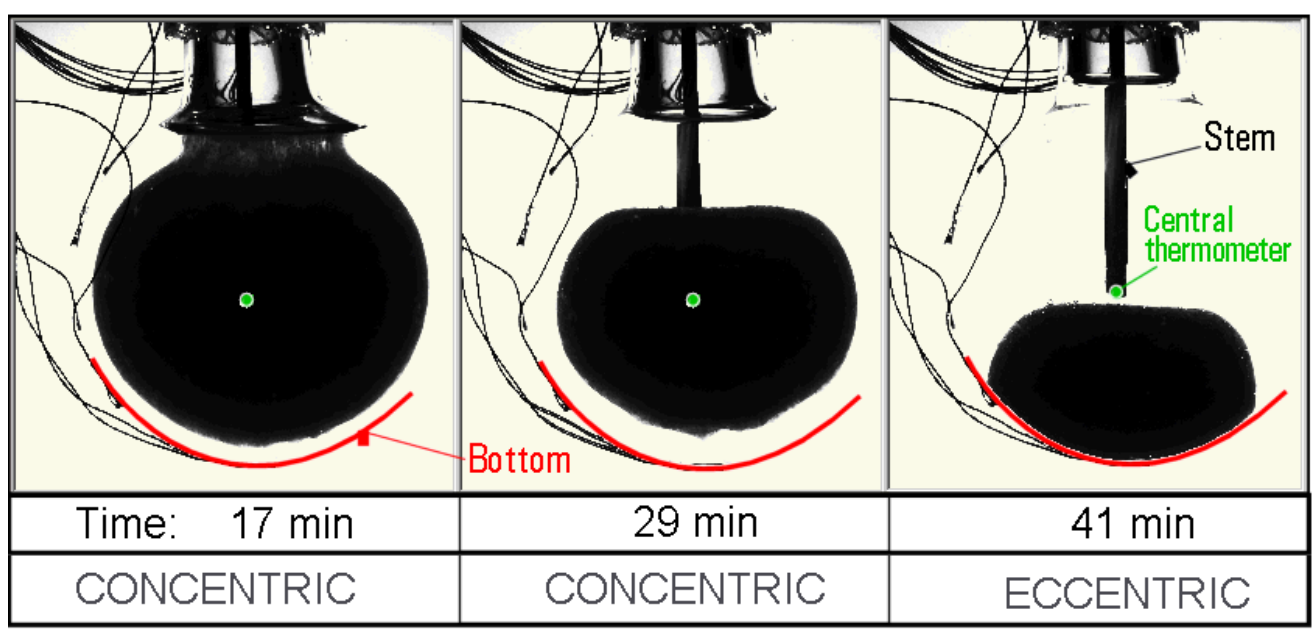

Fig. 19. The initially unexpected change of conditions during the experiment. The failure of the n-eicosane solid body to stay on the stem was initially considered a collapse of the whole experiment (which was too much time-consuming to be repeated). The gradually melted n-eicosane solid body was held from top on the vertical stem. At left, after 17 minutes past the beginning of the melting process, the solid body is still on the stem so that the liquid space (invisible due to the transparency of both gla ss and melt) is concentric. After added increment of $\mathbf{1 2}$ minutes (the central photograph) came the crisis: the body separated from the stem. Later, after another 12 minutes time increment (i.e. at 41 minutes from the start of the process), shown at right, the separated body sits on the shell bottom so that the thermometer initially positioned in the centre of the solid became exposed to the liquid phase. Of course, in the later stages the solid body ceases to be concentric and spherical.

for a quite long time, 16 hours, at a rather lpow temperature $25.315^{\circ} \mathrm{C}-$ i.e. substantially below the temperature if melting $\mathrm{T}_{\mathrm{m}}$. At the beginning of the test, the flask with the solidified n-eicosane body was submerged into the water in the rectangular test vessel and the the image Nr. 1 (Fig. 16) was taken. This instant was recorded as the starting time $t=0$, at which the flask contents was completely opaque. The subsequent melting experiment then lasted 70 minutes, with photographs taken every minute.

Unexpectedly, after about 37 minutes past the start of the image collecting, the solid n-eicosane body separated from the stem and dropped to the shell bottom — Fig. 19. This exposed the thermometer probe \#101

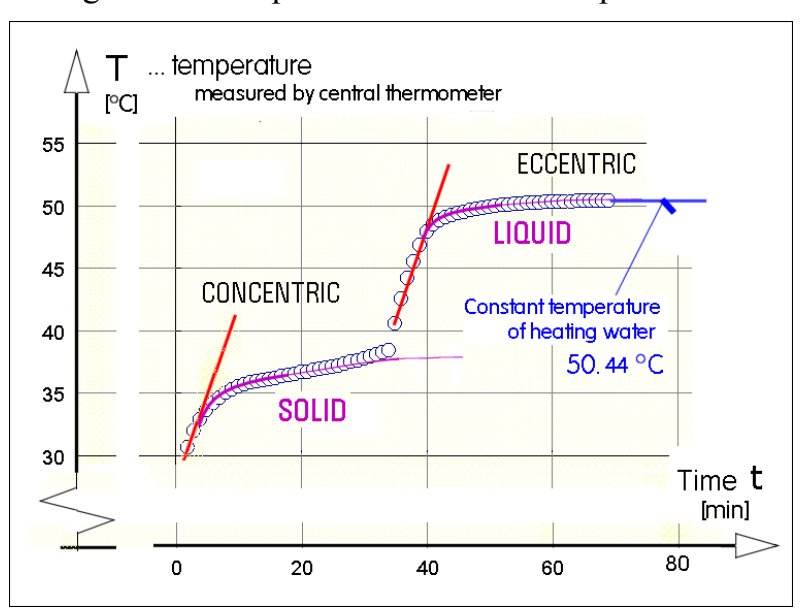

Fig. 19. Time dependence of the temperature $T_{101}$ measured by the resistor thermometer probe initially positioned in the centre of the n-eicosane solid body, at the tip of the vertical stem.

to the molten liquid which was warmer than the temperature inside the solid n-eicosane body. The consequent change of the temperature measured by this probe is presented in the dependence plotted in Fig. 20 showing clearly the character of the measured temperature. The two parallel large-slope red lines in Fig. 19 (for the concentric and eccentric configurations) are indicative of the temperature variation in the melting body - the unbalance from the constant temperature of melting and the gradual heating of the components of test setup. At the instant of the separation the conditions at the temperature probe \#101 changed from the initially concentric configuration (cf Fig. 6) into the eccentric ones and this provided the thermal difference references in the definition of the Rayleighh, Grasshoff, as well as Stefan numbers.

\subsection{The semi-logarithmic law}

In ref. [3] was found useful to plot the solidification data from [11] in semi-logarithmic co-ordinates. The data points there were melt fraction on the vertical linear co-ordinate and the time converted into the product of the Fourier and Stefan numbers on the logarithmic horizontal co-ordinate. The data points indicated an interesting universality for different sample sizes (spherical shell diameters) and, even more interestingly, they exhibited over a large part of the process a capability of being fitted by the straight line.

The analogous data were now accumulated also in the discussed experiment of the melting. The temperature difference $\Delta \mathrm{T}$ in the expressions presented in Fig. 21 was taken as the vertical difference between the solid and liquid part of the measured temperature history presented in Fig. 19. The magnitude of the instantaneous melt volume fraction was computed using the contours of the photographed solid bodies applied in the second central theorem of Pappus of Alexandria [14]. Of course, this was originally experimental run - the one presented in Fig. 20. 


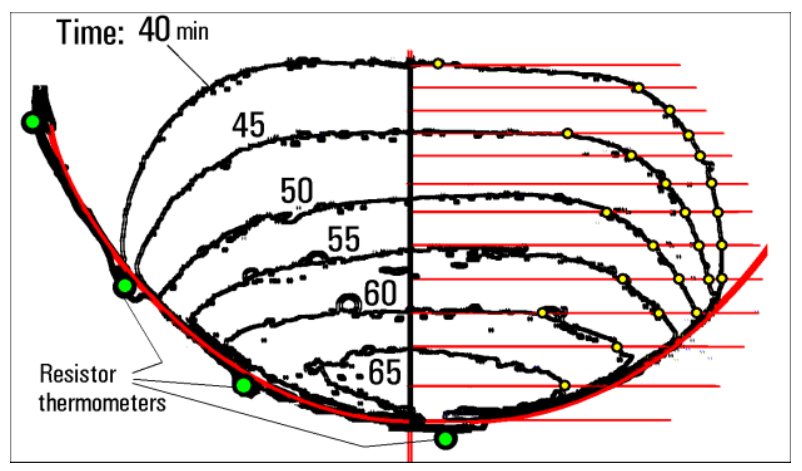

Fig. 20. Outer shapes of the solid n-eicosane inside the $64.1 \mathrm{~mm}$ outer diameter glass flask at the time instants after the separation from the stem \{cf. Figs. 6 and 18). The auxiliary horizontal red lines in the right-hand side of the picture were used to identify the surface points for calculating of the melted body shapes in the photographs.

The graphical presentation shown in Fig. 22 has, however, shown no expected discontinuity. The nondimensionalisation of elapsed time into the product of the Fourier and Stefan numbers was performed using the expressions in Fig. 21. At the initial, small times there is the transition regime strongly dependent upon the initial conditions, but later on, in the substantial part of diagram in Fig. 21, there is the regime of settled equilibrium in which the volume of the remaining solid is governed by the semi-logarithmic law.

\subsection{Indirect indications of unsteady streaming}

When the accumulated images of the solid body contours, such as those presented in Fig. 29 and here anew in Fig. 23, were inspected they show different sloped of the linear fits in the neighbourhoods of the vertical axis. Not only the slope values are not identical, but they do vary even in positive or negative values.

The obvious explanation is in oscillatory motion of the n-eicosane solid body. These motions may be driven by quite small horizontal forces - because

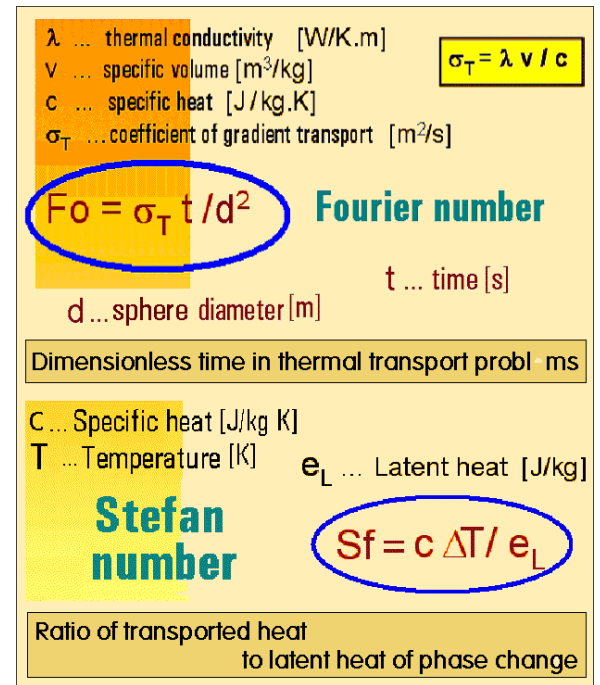

Fig. 21. Dimensionless parameters Fo and Sf. Their product Fo.Sf, was expected - as discussed in [3] - to be of fundamental importance in the search for the universal law of the melting process.

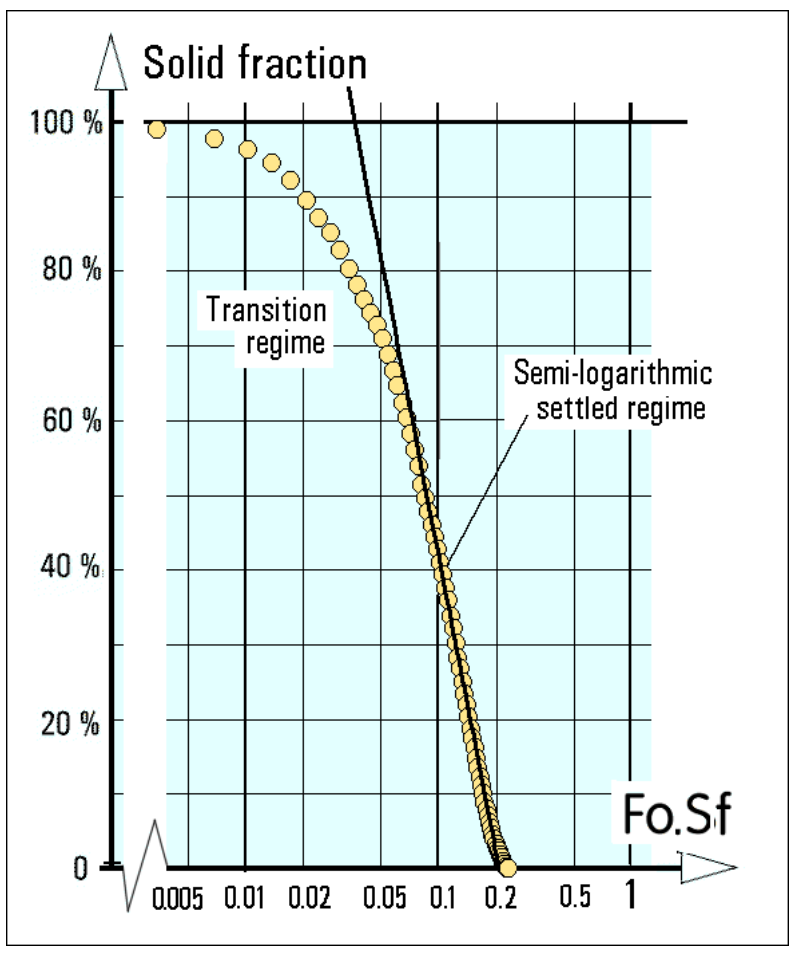

Fig. 22. Time history of melting the n-eicosane solid in the spherical capsule, evaluated from photographs of the surface of the solid (as they are seen in Fig. 20). The time scale is converted into the relative coordinates by evaluating Fourier number Fo and Stefan number Sf. according to Fig. 21. In the substantial part of this diagram, in the settled equilibrium regime, the volume of the remaining solid is governed by the semi-logarithmic law.

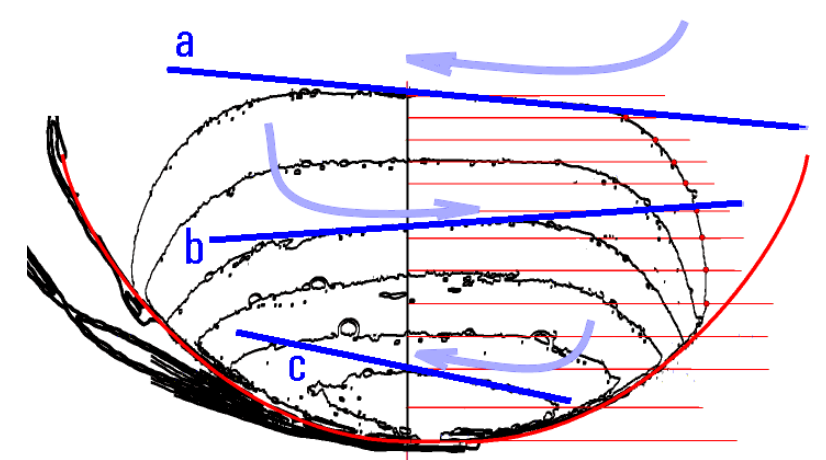

Fig. 23. Detailed views of the solid-body photographs show existence of very slow oscillatory motions. The top surface of the still solid (not yet molten) n-eicosane body are here fitted by straight lines. It is immediately apparent that the these lines (in blue colours) exhibit different slopes at different instants of time. Note in particular the slopes a, b, and c.

of the small curvature radius of the solid body bottom, sitting inside a "bowl" at the bottom of the shell, with its larger radius. Such horizontal forces may be generated by sideways components of the flows, in Fig. 23 indicated by the blue arrows. Another obvious demonstrations of the inclined body variation are presented in Fig. 24, within the last moments of the solid body existence. The red contour slopes downwards to the right - while the blue contour is 
sloped upwards. Of course, these indications of what is probably oscillation are indirect because the one minute intervals between taken photographs ale too large and cannot agree with the oscillation period. To resolve the enigma, it will be necessary to assemble images for a fast running camera.

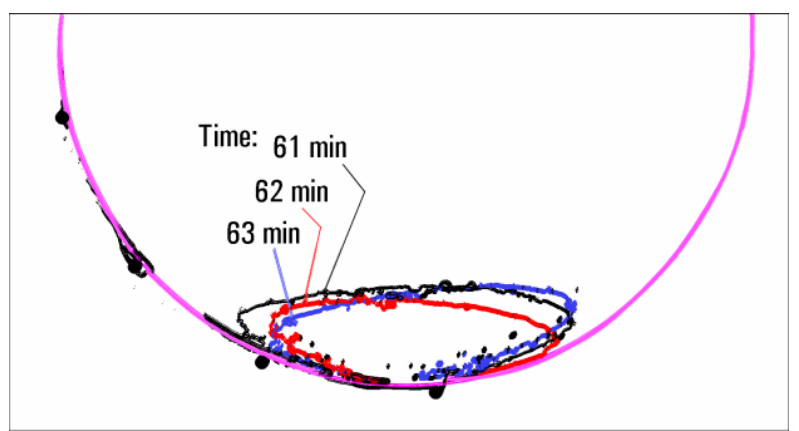

Fig. 24. Contours of photographed melted body in the last minutes of the melting process. The body obviously responds by the oscillatory motions to the unsteady convective flows present in the liquid phase.

\section{Conclusions}

Extreme climatic conditions, in particular those with intensive hot weather, are not only inconvenient but actually dangerous to health - especially if persons like soldiers in desert are required to be physically active $[20,21]$. A solution is expected in development of garments made from cooling "smart" textiles. The initially pursued version with Brayton cycle cannot be miniaturised to practically useful scales. It is replaced by having distributed in the textile small capsules filled with material melting near to the normal temperature of human body and employing for storage the heat of melting.

The paper consists of two parts. The first one accumulates information from literature about the processes that have to be clarified for understand the processes taking place in the capsules. Existing publications investigated heat transfer to capsules but expected invariant spherical geometry. The changes caused by the melting complicate the problem substantially. Also missing so far is the information on possible internal convection streaming in the varied geometry. The second part of the paper then presents preliminary results obtained from the first experiments with a scaled-up capsule model.

Authors were supported by research grant Nr. 17-08218S obtained from the GACR Czech Republic Grant Agency - and also by institutional support RVO: 61388998 .

\section{References}

1. V. Tesař, Chapter 6.1.2 "Cooling garments and the problem of portable power units", pp. 311-319, in monograph Pressure-Driven Microfluidics, Artech House Publishers, Norwood, USA, 2007

2. C. H. Oh, R. L. Moore, Nuclear technology, 149, 324, 2017

3. V. Tesar̆, EPJ Web of Conferences, 180, 02108, 2018

4. E.H. Bishop, L.R. Mack, J.A. Scanlan, Int. J. Heat Mass Transfer, 9, 649, 1966

5. P. Teertstra, M. Yovanovih, J.R. Culham. J. Heat Transfer 128, 580, 2006

6. S. H. Yin et al., Int. J. Heat Mass Transfer 16, 1785, 1973

7. Zoelly R.., Dissertation, ETH Zürich 1915

8. Schwerin A., ZAMM, 2, 81, 1922

9. von Kármán T., Tsien H. S., Journal of Aeronautical Sciences, . 7, 1939

10. Krenke M. A., Kiernan T. J., AIAA Journal, 1, 2855, 1963

11. E. Assis, G. Ziskind, Letan R., Journal of Heat Transfer 131, 024502-1, 2009

12. C. Vélez, M. Khayet, J. M. Ortiz de Zárate, Applied energy 143, p. 394, 2015

13. M. Akanksha, A. Shukla, A. Sharma, Resonance, 20 532,2015

14 A. Jones, "Pappus of Alexandria", Book 7 of the collection, Springer New York, 1986

15. D. W. Yarbrough D.W., Kuan C-N.,Proceedings of the 17th International Thermal Conductivity Conference, Gaithersburg, Maryland, USA, p 265, 1981

16. N. S. Dhaidan, Journal of Renewable and Sustainable Energy, 9, 054101, 2017

17. J. M. Khodadadi and Y. Zhang, Internat. Journ. of Heat and Mass Transfer, 44, p. 1605, 2001

18. S. A. Aksoy, et al., The Journal of the Textile Institute, 108, 1, 2017

19. A. Shaid, et al., Applied Thermal Engineering, 107, p. 602, 2016

20. E.F. Adolph, Physiology of man in the desert, New York Interscience Publishers, 1947

21. Y. Kuno, Human perspiration, Springfield IL, C. C. Thomas 1956, 